\title{
Involvement of oxidative stress in patients of gout and antioxidant effect of allopurinol
}

\author{
Chetan R Acharya, Abhinav K Sharma, ND Kantharia \\ Department of Pharmacology, Government Medical College, Surat, Gujarat, India. \\ Correspondence to: Abhinav K Sharma, E-mail: ramvaidehi@gmail.com
}

Received October 3, 2014. Accepted October 10, 2014

\section{Abstract}

Background: Various experimental, epidemiological, and clinical studies have shown that hyperuricemia is associated with development of hypertension, visceral obesity, insulin resistance, dyslipidemia, type 2 diabetes, kidney diseases, and cardiovascular and cerebrovascular events. Whether hyperuricemia is a cause or effect is a debatable subject. Although the pathogenesis of these diseases is complex and incompletely understood, it is clear that oxidative stress and damage to proteins and lipids is common for all of them.

Objective: To determine whether hyperuricemia causes oxidative stress in patients with gout, and to evaluate the effect of allopurinol treatment on oxidative stress after 1 and 3 months in these patients.

Materials and Methods: Patients with newly diagnosed gout $(n=30)$ and healthy age- and sex-matched volunteers $(n=30)$ were enrolled in the study. They were treated with $100-\mathrm{mg}$ allopurinol 8 hourly in a day for 3 months. Serum uric acid levels and levels of oxidative stress such as serum malondialdehyde (MDA), erythrocyte catalase (CAT), and erythrocyte superoxide dismutase (SOD) were measured before treatment, and after 1 and 3 months of treatment. Pretreatment levels of all the parameters were compared in both the groups using unpaired $t$-test. The parameters were also analyzed in test group at 0,1 , and 3 months of treatment using repeated-measures analysis of variance test.

Results: Pretreatment levels of serum uric acid and MDA were significantly higher in patients as compared to control group $(P<0.01)$. They were significantly reduced after $1(P<0.05)$ and 3 months $(P<0.01)$ of treatment with allopurinol. Pretreatment levels of CAT and SOD were significantly lower in patients as compared to control group $(P<0.01)$; they were increased significantly after $1(P<0.01)$ and 3 months $(P<0.01)$ of treatment with allopurinol.

Conclusion: From this study, it can be suggested that hyperuricemia causes oxidative stress in patients with gout. Besides the main benefit of lowering serum uric acid level, allopurinol also decreases oxidative stress in patients with gout.

KEY WORDS: Gout, hyperuricemia, oxidative stress, allopurinol

\section{Introduction}

Gout is a common crystal-induced arthropathy. It is caused by a group of heterogenous disorders that lead to either overproduction or underexcretion of uric acid or both. When serum uric acid concentration reaches its threshold for solubility $(6.8 \mathrm{mg} / \mathrm{dL})$, it starts to get deposited in the form of monosodium urate monohydrate crystals in joint spaces and

\begin{tabular}{|l|l|}
\hline Website: htp://www.jmsph.com & Access this article online \\
\hline DOl: 10.5455/jimsph.2015.0310201435 & \\
\hline
\end{tabular}

other tissues. Deposition of these crystals in joint space leads to neutrophil activation that causes synovial inflammation in short term and joint deformity in long term. ${ }^{[1,2]}$

Various experimental, epidemiological, and clinical studies have shown that hyperuricemia is associated and in many cases predicts development of hypertension, visceral obesity, insulin resistance, dyslipidemia, type 2 diabetes, kidney diseases, and cardiovascular and cerebrovascular events. Whether hyperuricemia is a cause or an effect is a debatable subject. Although the pathogenesis of these diseases is complex and incompletely understood, it is clear that oxidative stress and damage to proteins and lipids is common for all of them. ${ }^{[3]}$

Allopurinol is an inhibitor of xanthine oxidase enzyme, which catalyzes formation of uric acid from xanthine. It is indicated for treatment of chronic gout and for recurrent attacks of acute gout. ${ }^{[4]}$ Besides its hypouricemic effect, there are direct and indirect evidences of antioxidant effect of allopurinol. 
It was shown to scavenge superoxide anion and hydroxyl radicals in in vitro and experimental studies, whereas it improves endothelial dysfunction caused by oxidative stress in patients of congestive heart failure, type 2 diabetes, and coronary artery disease, and in smokers. ${ }^{[5]}$

Considering the above-mentioned facts, relationship among hyperuricemia, allopurinol, and oxidative stress is complex. There are not enough evidences regarding effect of hyperuricemia on oxidative stress in humans and antioxidant effect of allopurinol in patients with gout. Thus, this study was conducted with the objective to evaluate the effect of hyperuricemia on oxidative stress in patients with gout. Another objective was to evaluate whether allopurinol has any antioxidant effect in these patients.

\section{Materials and Methods}

This study was conducted at Government Medical College and New Civil Hospital, Surat, Gujarat, India, from June 2011 to May 2012. The study protocol was approved by the institutional ethics committee (MCS/STU/Ethics Approval/ 18750/2010).

\section{Subjects}

The individuals were divided into two groups: Patient group and control group.

Patient group: Thirty patients with gout (25 men and 5 women), eligible for allopurinol treatment, were enrolled after taking informed consent. They were diagnosed by preliminary criteria for diagnosis of gout given by the American College of Rheumatology, 1977. Only those patients who were previously not treated with any hypouricemic drug were enrolled. Mean age for study group was $42.17 \pm 12.54$ years.

Control group: Thirty age- and sex-matched healthy volunteers ( 25 men and 5 women) were enrolled after taking informed consent. Mean age for study group was $43.87 \pm$ 12.44 years.

Subjects with a history of cardiovascular diseases, diabetes, renal diseases; patients with active infections; patients on medications for diseases other than gout; and smokers and alcoholics were excluded from the study. Subjects were instructed not to take any nutritional supplements during the study period.

\section{Treatment Schedule}

The patients with gout were treated by tablet $100-\mathrm{mg}$ allopurinol three times daily for 3 months. The drug was procured from Central Medical Store, New Civil Hospital, Surat, Gujarat, India.

\section{Sample Collection and Biochemical Estimation}

Blood samples of control group and that of study group at the time of enrolment, after 1 month, and after 3 months of allopurinol treatment were collected. Tests for serum uric acid, serum malondialdehyde (MDA), erythrocyte superoxide dismutase (SOD), and erythrocyte catalase were conducted.

For oxidative stress parameters, $5 \mathrm{~mL}$ venous blood sample was collected in sodium EDTA $(1 \mathrm{mg} / \mathrm{mL})$ tubes and immediately subjected to biochemical analysis. The blood was centrifuged at $3000 \mathrm{rpm}$ for $15 \mathrm{~min}$ and then plasma separated was used for serum MDA estimation. The cells were washed with normal saline three times and RBCs were used for the estimation of SOD and catalase. RBCs $(0.5 \mathrm{~mL})$ were subjected to lysis by adding 3-mL ice-cold distilled water. Hemoglobin level in the hemolysate was measured using automated cell counter and hemoglobinometer machine in the Department of Pathology, New Civil Hospital, Surat, Gujarat. Hemoglobin in the hemolysate was precipitated by adding $1 \mathrm{~mL}$ ethanol and $0.6 \mathrm{~mL}$ chloroform. The mixture was mixed thoroughly on the vortex and then centrifuged at $3000 \mathrm{rpm}$ for $15 \mathrm{~min}$. Supernatant was used for estimation of erythrocyte SOD and erythrocyte catalase activity.

\section{Estimation of Serum Malondialdehyde}

The thiobarbituric acid reactive substance levels were estimated per the spectrophotometric method described by Ohkawa et al. ${ }^{[6]}$ Pink chromogen produced by reaction of thiobarbituric acid with MDA was measured at $532 \mathrm{~nm}$. The values are expressed as $\mathrm{nM} / \mathrm{mL}$ of plasma.

\section{Estimation of Erythrocyte Superoxide Dismutase}

The activity of SOD in erythrocytes was determined using the method described by Marklund and Marklund ${ }^{[7]}$ with some modifications as described by Nandi and Chatterjee. ${ }^{[8]}$ Pyrogallol is autoxidized rapidly in alkaline solution in the presence of superoxide anion to intermediate products and the solution first becomes yellow-brown with a maximum absorbance at $420 \mathrm{~nm}$. The rate of inhibition of pyrogallol autoxidation brought about by SOD is used for determination of the enzyme. The values are expressed as $\mathrm{U} / \mathrm{g}$ of hemoglobin.

\section{Estimation of Erythrocyte Catalase}

Catalase was measured using the method described by Sinha. ${ }^{[9]}$ Catalase acts on hydrogen peroxide $\left(\mathrm{H}_{2} \mathrm{O}_{2}\right)$ to release molecular oxygen. When potassium dichromate is added to this reaction mixture, activity of catalase is destroyed and any hydrogen peroxide that has not been split by the catalase reacts with dichromate to give a blue precipitate of perchromic acid. This unstable precipitate is then decomposed by heating to give the green solution and its absorbance is measured at $570 \mathrm{~nm}$. The values are expressed as $\mu \mathrm{mol}$ of $\mathrm{H}_{2} \mathrm{O}_{2}$ consumed/min/mg of hemoglobin.

All chemicals were purchased from ACS Ltd.

\section{Statistical Analysis}

All the values are expressed as mean \pm SD. The levels of serum uric acid and oxidative stress parameters of control group and patient group at the time of enrolment was 
compared using unpaired $t$-test. The comparison of serum uric acid level and oxidative stress parameters at the time of enrolment, after 1 month of allopurinol treatment, and after 3 months of allopurinol treatment in patients with gout was performed using repeated-measures analysis of variance test. $P$ value $<0.05$ was taken as significance level. All statistical analysis was conducted using SPSS software, version 16.0, for Windows.

\section{Results}

Results are shown in Table1. All the values are expressed as mean $\pm S D$. Serum uric acid levels are significantly higher in patient group than in control group at the time of enrolment, which decreased significantly after allopurinol treatment. A significant increase in plasma MDA level whereas a significant decrease in SOD and catalase levels were observed in patient group in comparison to control group at the time of enrolment, which suggests significant increase in oxidative stress. A significant decrease in serum MDA level whereas a significant increase in SOD and catalase levels were observed in patient group with allopurinol treatment after 1 and 3 months, which suggests significant decrease in oxidative stress after allopurinol treatment.

\section{Serum Uric Acid Level}

The level of serum uric acid in patients with gout was significantly higher than that in control group at the time of enrolment $(P<0.01)$. Treatment with allopurinol decreases serum uric acid level significantly after 1 and 3 months $(P<0.01)$

\section{Lipid Peroxidation}

The levels of serum MDA, a marker of lipid peroxidation, were significantly higher for the patient group than that for the control group at the time of enrolment $(P<0.01)$. In the patient group, treatment with allopurinol decreased MDA level significantly after $1(P<0.05)$ and 3 months $(P<0.01)$, whereas no significant difference in MDA level after 1 and 3 months of allopurinol treatment was observed in control group.

\section{Antioxidant Enzymes}

SOD and catalase are antioxidant enzymes. Erythrocyte SOD and erythrocyte catalase levels in study group are significantly lower than those in control group at the time of enrolment $(P<0.01)$. Treatment with allopurinol increases erythrocyte SOD and erythrocyte catalase levels significantly after 1 and 3 months $(P<0.01)$. No significant difference in SOD level after 1 and 3 months of allopurinol treatment was observed.

\section{Discussion}

Results of this study showed increased oxidative stress in patients with newly diagnosed gout at the time of enrolment in comparison to healthy subjects. As other factors known to cause increase oxidative stress were absent, it may be suggested that hyperuricemia causes oxidative stress in patients with gout. The oxidative stress was reduced by allopurinol treatment (100 mg every 8 hourly) at 1 month and the effect was same for 3 months of treatment.

Our results are in line with the findings of oxidative stress present in various experimental models of hyperuricemia and antioxidant effect of allopurinol in them. ${ }^{[10,11]}$

Association of hyperuricemia with hypertension, visceral obesity, type 2 diabetes, insulin resistance, renal diseases, and cardiovascular and cerebrovascular events can be explained on the basis of the results of this study. As hyperuricemia causes oxidative stress and interrelated trio of vascular inflammation, oxidative stress, and endothelial dysfunction, which lead to oxidative damage of lipids and proteins, is the principal cause in pathology of all the abovementioned conditions. ${ }^{[3]}$ Further, there are evidences that cardiovascular mortality and all-cause mortality in these patients are significantly higher than those in patients with normal serum uric acid levels. ${ }^{[12,13]}$ Allopurinol also improved endothelial dysfunction in some of the above conditions. This suggests that association of hyperuricemia with abovementioned conditions is not merely a response of body to increased oxidative stress but hyperuricemia itself causes oxidative stress and by virtue of this has some role in the pathology of them. This is also supported by findings of LIFE $^{[14]}$ and GREACE ${ }^{[15]}$ trials that showed that beneficial role

Table 1: Serum uric acid, serum malondialdehyde, erythrocyte SOD, and erythrocyte catalase level in control and patient groups

\begin{tabular}{|c|c|c|c|c|}
\hline & \multirow[t]{2}{*}{ Control group $(n=30)$} & \multicolumn{3}{|c|}{ Patient group $(n=30)$} \\
\hline & & At enrolment & After 1 month & After 3 months \\
\hline Serum uric acid (mg/dL) & $3.8 \pm 0.8$ & $6.9 \pm 1.6^{*}$ & $5.1 \pm 1.3^{\#}$ & $5.0 \pm 1^{\#}$ \\
\hline Serum MDA $(\mathrm{nM} / \mathrm{mL})$ & $2.68 \pm 0.69$ & $5.73 \pm 1.55^{\star}$ & $4.97 \pm 0.76^{\# \#}$ & $4.87 \pm 0.82^{\#}$ \\
\hline $\mathrm{SOD}(\mathrm{U} / \mathrm{g}$ of $\mathrm{Hb})$ & $1192 \pm 149$ & $1059 \pm 149^{*}$ & $1155 \pm 167^{\#}$ & $1165 \pm 163^{\#}$ \\
\hline Catalase $\left(\mu \mathrm{M}\right.$ of $\mathrm{H}_{2} \mathrm{O}_{2}$ consumed $\left./ \mathrm{mg} / \mathrm{min}\right)$ & $2273 \pm 248$ & $1988 \pm 149^{*}$ & $2220 \pm 163^{\#}$ & $2216 \pm 167^{\#}$ \\
\hline
\end{tabular}

${ }^{*} P<0.01$ in comparison to control group; ${ }^{\#} P<0.01$ in comparison to study group at enrolment; ${ }^{\# \#} P<0.05$ in comparison to study group at enrolment. 
of losartan and atorvastatin, respectively, was partly due to their uric acid-lowering activity in associated cardiovascular conditions. As scope of this study was limited in terms of time frame and study population, the effect of hyperuricemia on cardiovascular parameters and events was not studied, but presence of oxidative stress in the study population points toward development of such events in long term as evidenced by literature.

Under normal physiological conditions, uric acid acts as an antioxidant in plasma. ${ }^{[16]}$ Our findings are in contrast to this concept. This can be explained on the basis of phenomenon of "antioxidant-prooxidant urate redox shuttle" in which an antioxidant uric acid acts as prooxidant under certain conditions, importance of which is when serum uric acid level lies in upper two thirds of normal range, that is, more than $4 \mathrm{mg} / \mathrm{dL}$ and out of range, that is, $6 \mathrm{mg} / \mathrm{dL}$ for women and $6.5 \mathrm{mg} / \mathrm{dL}$ for men. ${ }^{[17]}$

The exact mechanism for hyperuriemia-induced oxidative stress and antioxidant effect of allopurinol was not explored in this study, but certain hypothesis can be made based on the earlier works conducted in this field. Uric acid by activating inflammatory pathways inducing vascular smooth-muscle-cell proliferation and increasing low-density lipoprotein cholesterol oxidation starts the process of atherosclerosis in vascular walls. In these atherosclerotic plaques, due to combined effect of ischemia necrosis and apoptosis, there occurs increased supply of purine nucleic acids and increased activity of xanthine oxidase enzyme. This results in overproduction of uric acid at that site and simultaneous production of superoxide anion that leads to oxidative stress. Increased concentration of uric acid causes uncoupling of endothelial NO synthase that further increases superoxide radical production. Owing to inflammation and angiogenesis at atherosclerotic plaques, there is frequent rupture of vasa vasorum that releases iron and copper ions in the medium. Superoxide radicals in the presence of these ions form hydroxyl radicals through Fenton and Haber-Wiess reactions, which further propagate oxidative damage and increase oxidative stress. Uric acid itself in these favorable conditions increases ROS production and lipid peroxidation in human adipocyte through activation of NADPH oxidase. ${ }^{[17,18]}$

Allopurinol may decrease oxidative stress by decreasing serum uric acid and resultant harmful effects as described earlier. It can also decrease oxidative stress by inhibiting xanthine oxidase. Xanthine oxidase, besides its function in purine metabolism, is also involved in superoxide anion generation. ${ }^{[19]}$ Allopurinol can directly scavenge superoxide anion and hydroxyl radical, thus decreasing oxidative stress.

\section{Conclusion}

From the findings of this study, it can be suggested that hyperuricemia, by increasing oxygen free radical production and resultant oxidative stress, causes tissue damage in the patients with gout. Allopurinol besides its serum uric acid-lowering effect provides an additional advantage of lowering oxidative stress in these patients.

\section{References}

1. Biyani MR, Aiya MN, Velingkar VS. Gout-a review. Indian J Pharm Sci. 2005;67(5):513-22.

2. Longo DL, Kasper DL, Jameson JL, Anthony SF, Hauser SL, Loscalzo J. Harrison's Principles of Internal Medicine, 18th edn. New York: McGraw-Hill, 2012.

3. Sautin $Y Y$, Johnson RJ. Uric acid: the oxidant-antioxidant paradox. Nucleosides Nucleotides Nucleic Acids. 2008;27(6): 608-19.

4. Brunton LL, Chabner BA, Knollman BC. Goodman \& Gilman's the Pharmacological Basis of Therapeutics, 12th edn. New York: McGraw-Hill, 2011.

5. Pacher P, Nivorozhkin A, Szabo C. Therapeutic effects of xanthine oxidase inhibitors: renaissance half a century after the discovery of allopurinol. Phrmacol Rev. 2006(58):87-114.

6. Ohkawa H, Ohishi N, Yagi K. Assay for lipid peroxides in animal tissues by thiobarbituric acid reaction. Anal Biochem. 1979;95: 351-8.

7. Marklund S, Marklund G. Involvement of superoxide anion radical in the autoxidation of pyrogallol and a convenient assay of superoxide dismutase. Eur J Biochem. 1974;47:469-76.

8. Nandi A, Chatterjee IB. Assay of superoxide dismutase activity in animal tissue. J Biosci. 1988;13:305-15.

9. Sinha AK. Colorimetric assay of catalase. Anal Biochem. 1972;47:389-94.

10. Watanabe S, Kiyama F, Sakamaki A, Yoshidaa T, Fukui T. Cerebral oxidative stress and mitochondrial dysfunction in oxonate-induced hyperuricemic mice. J Health Sci. 2006;52(6):730-7.

11. Watanabe S, Tajima $\mathrm{Y}$, Yamaguchi T, Fukui T. Potassium bromate-induced hyperuricemia stimulates acute kidney damage and oxidative stress. J Health Sci. 2004;50(6)647-53.

12. Tomita M, Mizuno S, Yamanaka H, Hosoda $Y$, Sakuma K, Matuoka $\mathrm{Y}$, et al. Does hyperuricemia affect mortality? A prospective cohort study of Japanese male workers. J Epidemiol. 2000;10:403-9.

13. Berg $A H$, Lin $\mathrm{Y}$, Lisanti MP, Scherer PE. Adipocyte differentiation induces dynamic changes in NF- $\kappa \beta$ expression and activity. Am J Physiol Endocrinol Metab. 2004;287:E1178-88.

14. Høiggen A, Alderman M, Kjeldsen SE, Julius S, Devereux RB, De Faire $U$, et al. The impact of serum uric acid on cardiovascular outcomes in the LIFE study. Kidney Int. 2004;65:1041-9.

15. Athyros VG, Elisaf $M$, Papageorgiou AA, Symeonidis $A N$, Pehlivandis AN, Bouloukos VI, et al. Effect of statins versus untreated dyslipidaemia on serum uric acid levels in patients with coronary heart disease; a subgroup analysis of the GREek Atorvastatin and Coronary-heart-disease Evaluation (GREACE) study. Am J Kidney Dis. 2004;43:589-99.

16. Ames $B N$, Cathcart $R$, Schwiers $E$, Hochstein P. Uric acid provides an antioxidant defense in humans against oxidant- and radical-caused aging and cancer: a hypothesis. Proc Natl Acad Sci USA. 1981;78:6858-62.

17. Hayden MR, Tyagi SC. Uric acid: a new look at an old risk marker for cardiovascular disease, metabolic syndrome, and type 2 diabetes mellitus: The urate redox shuttle. Nutr Metab (Lond). 2004;1(1):10. 
18. Sautin YY, Nakagawa T, Zharikov S, Johnson RJ. Adverse effects of the classical antioxidant uric acid in adipocytes: $\mathrm{NADPH}$ oxidase-mediated oxidative/nitrosative stress. Am J Physiol Cell Physiol. 2007;293:C584-96.

19. Sanders SA, Massey V. The thermodynamics of xanthine oxidoreductase catalysis. Antioxid Redox Signal. 1999;1: $371-9$.
How to cite this article: Acharya CR, Sharma AK, Kantharia ND. Involvement of oxidative stress in patients of gout and antioxidant effect of allopurinol. Int $\mathrm{J}$ Med Sci Public Health 2015;4:168-172

Source of Support: Nil, Conflict of Interest: None declared. 